\title{
GENERALIZED FUNCTIONALS OF BROWNIAN MOTION
}

\author{
N.U. AHMED \\ University of Ottawa \\ Department of Electrical Engineering and \\ Department of Mathematics \\ Ottawa, Ontario, CANADA K1N $6 N 5$
}

(Received March 1993; revised June, 1993)

\begin{abstract}
In this paper we discuss some recent developments in the theory of generalized functionals of Brownian motion. First we give a brief summary of the Wiener-Ito multiple Integrals. We discuss some of their basic properties, and related functional analysis on Wiener measure space. then we discuss the generalized functionals constructed by Hida. The generalized functionals of Hida are based on $L_{2}$-Sobolev spaces, thereby, admitting only $H^{s}, s \in R$ valued kernels in the multiple stochastic integrals. These functionals are much more general than the classical Wiener-Ito class. The more recent development, due to the author, introduces a much more broad class of generalized functionals which are based on $L_{p}$-Sobolev spaces admitting kernels from the spaces $W^{p, s}, s \in R$. This allows analysis of a very broad class of nonlinear functionals of Brownian motion, which can not be handled by either the Wiener-Ito class or the Hida class. For $s \leq 0$, they represent generalized functionals on the Wiener measure space like Schwarz distributions on finite dimensional spaces. In this paper we also introduce some further generalizations, and construct a locally convex topological vector space of generalized functionals. We also present some discussion on the applications of these results.
\end{abstract}

Key words: Generalized Functionals, Brownian Motion, Stochastic Distributions, Wiener Measure, Stochastic Differential Equations.

AMS (MOS) subject classifications: $46 \mathrm{~F} 25,60 \mathrm{G} 20,60 \mathrm{H} 07,60 \mathrm{H} 15$.

\section{Introduction}

It is known that Volterra series can be used to approximate any continuous functional on a Banach space. Similarly it was Wiener who first proved that any $L_{2}$ functional of Brownian motion can be approximated in the limit in the mean by sums of multiple stochastic integrals. We shall call these as regular functionals of Brownian motion. Later in the eighties Hida introduced an interesting class of generalized functionals of the Wiener process by use of a notion of Fourier transform on the dual of a Nuclear space which supports white noise measure. This generalization is significant and applies to problems where the regular functionals are insufficient. In fact white noise itself is a generalized functional of Brownian motion and does not belong to the class $L_{2}$ of regular functionals. We shall discuss this later in the paper. In the early eighties [5] the author introduced a class of generalized functionals of white noise which cover those of 
Hida as special cases. Further the method of construction used in the author's paper [5] is direct and simple and does not use the Fourier transform as employed by Hida. In any case both the models are instructive and will be discussed in the paper. Let $(\Omega, \mathscr{B}, P)$ be a complete probability space and $I \equiv(0, T)$ an open bounded interval in $R$ and $\{w(t), t \in I\}$ the standard Wiener process on $(\Omega, \mathfrak{B}, P)$ starting from the origin with probability one.

\section{Regular Functionals of Brownian Motion}

Let $\Omega=C_{0}(I)$ denote the canonical sample space where $C_{0}(I) \equiv\{x \in C(I): x(0)=0\}$, and let $\mathscr{B}$ denote the Borel $\sigma$-algebra of subsets of the set $\Omega$ and let $\mu$ denote the Wiener measure. The class of regular homogeneous functionals of Brownian motion of degree $n$ is defined by

$$
g_{n}(w) \equiv \int_{I^{n}} K_{n}\left(\tau_{1}, \tau_{2}, \ldots, \tau_{n}\right) d w\left(\tau_{1}\right) d w\left(\tau_{2}\right) \ldots d w\left(\tau_{n}\right)
$$

for any $K_{n} \in L_{2}\left(I^{n}\right)$ where $I^{n}$ denotes the Cartesian product of $n$-copies of $I$. These are the multiple Wiener integrals based on Brownian motion and are known as polynomial chaos of degree $n$. For each $K_{n} \in L_{2}\left(I^{n}\right)$, the functional (2.1) is well defined in the Ito-Wiener sense and belongs to $L_{2}(\Omega, \mathscr{B}, \mu)$. For each $n \in N_{+}$, let $\widehat{L}_{2}\left(I^{n}\right)$ denote the class of symmetric $L_{2}\left(I^{n}\right)$ kernels. In order to emphasize the dependence of $g_{n}$ on the kernels $K_{n}$, we shall often write $g_{n}(w)$ as $g_{n}\left(K_{n}, w\right)$. We state some basic properties of these functionals.

$(P 1)$ For each $K_{n} \in L_{2}\left(I^{n}\right), w \rightarrow g_{n}\left(K_{n}, w\right)$ is a $\mu$-measurable function on $\Omega$ and belongs to $L_{2}(\Omega, \Im, \mu)$.

(P2) For each $K_{n} \in \widehat{L}_{2}\left(I^{n}\right)$ and $L_{m} \in \widehat{L}_{2}\left(I^{m}\right)$

$$
\left\langle g_{n}\left(K_{n}, \cdot\right), g_{m}\left(L_{m}, \cdot\right)\right\rangle_{\mu} \equiv \int_{\Omega} g_{n} g_{m} d \mu=0, \text { for } m \neq n .
$$

(P3) For each $K_{n} \in \widehat{L}_{2}\left(I^{n}\right)$ and $L_{n} \in \widehat{L}_{2}\left(I^{n}\right)$

$$
\begin{aligned}
& \left\langle g_{n}\left(K_{n}, \cdot\right), g_{n}\left(L_{n}, \cdot\right)\right\rangle_{\mu} \equiv \int_{\Omega} g_{n}\left(K_{n}, w\right) g_{n}\left(L_{n}, w\right) d \mu(w) \\
& =n ! \int_{I^{n}} K_{n}\left(\tau_{1}, \tau_{2}, \ldots, \tau_{n}\right) L_{n}^{*}\left(\tau_{1}, \tau_{2}, \ldots, \tau_{n}\right) d \tau_{1} d \tau_{2} \ldots d \tau_{n} .
\end{aligned}
$$

(P4) The map $K_{n} \rightarrow g_{n}\left(K_{n}, \cdot\right)$ from $L_{2}\left(I^{n}\right)$ to $L_{2}(\Omega, \mathscr{B}, \mu)$ is linear and further it is continuous in the sense that whenever the sequence $K_{n}^{r} \stackrel{s}{\rightarrow} K_{n}^{0}$ in $L_{2}\left(I^{n}\right)$ as $r \rightarrow \infty$,

$$
g_{n}\left(K_{n}^{r}, \cdot\right) \stackrel{s}{\rightarrow} g_{n}\left(K_{n}^{0}, \cdot\right) \text { in } L_{2}(\Omega, \mathfrak{B}, \mu)
$$

The most important properties are $(P 2)$ and $(P 3)$ and they state that the functionals $\left\{g_{n}\right\}$ are orthogonal in $L_{2}(\Omega, \Re, \mu)$. Further, it was proved by Wiener that they are complete in the class $L_{2}(\Omega, \mathscr{B}, \mu)$ (see also [4]). Hence they form a basis for the Hilbert space $L_{2}(\Omega, \mathscr{B}, \mu)$. Thus these functionals play a similar role as the sine cosine functions in the Fourier analysis of $L_{2}$ functions on finite dimensional spaces. Several years ago, the author proved some basic results on the Fourier analysis on Wiener measure space [1] such as Bessel's inequality, Parsevals equality, and Riesz-Fischer theorem. We state these results here without proof. For proof, the reader is referred to the original papers [1-4]. From now on, we shall suppress the $w$ variable and write $g_{n}\left(K_{n}\right)$ in place of $g_{n}\left(K_{n}, w\right)$. 
Lemma 2.1: For each $f \in L_{2}(\Omega, \mathscr{B}, \mu)$ and each $s \in N_{+}$, there exists a unique kernel $L_{s} \in \widehat{L}_{2}\left(I^{s}\right)$, with respect to the fundamental basis $\left\{g_{n}\right\}$, such that

$$
\left\langle f, g_{s}\left(K_{s}\right)\right\rangle_{\mu}=s ! \int_{I^{s}} L_{s}\left(\tau_{1}, \tau_{2}, \ldots, \tau_{s}\right) K_{s}^{*}\left(\tau_{1}, \tau_{2}, \ldots, \tau_{s}\right) d \tau_{1} d \tau_{2} \ldots d \tau_{s},
$$

for all $K_{s} \in L_{2}\left(I^{s}\right)$.

This result states that, given the fundamental set $\left\{g_{n}\right\}$, the Fourier-Wiener kernels for any functional $f \in L_{2}(\Omega, \mathfrak{B}, \mu)$ are uniquely determined by $f$ alone.

Next we present Parseval's inequality. For convenience of notation, we let $L_{2}\left(I^{0}\right)$ denote the scalars.

Theorem 2.2: Let $\left\{g_{n}\left(K_{n}\right)\right\}, K_{n} \in \widehat{L}_{2}\left(I^{n}\right)$ be the fundamental set as defined above. Then for each $f \in L_{2}(\Omega, \mathscr{B}, \mu)$,

$$
\langle f, f\rangle_{\mu} \equiv\|f\|^{2} \geq \sum_{s=0}^{s=n} s ! \int_{I^{s}}\left|L_{s}\left(\tau_{1}, \tau_{2}, \ldots, \tau_{s}\right)\right|^{2} d \tau_{1} d \tau_{2} \ldots d \tau_{s}, n \in N_{+},
$$

where $L_{2} \in \widehat{L}_{2}\left(I^{s}\right)$ are the Fourier-Wiener kernels uniquely determined by $f$ through the relation $(2.2)$.

Proof: For proof see [1].

As a consequence of this result one can assert that for a given function $f \in L_{2}(\Omega, \mathscr{B}, \mu)$ to be approximated in the limit in the mean by a suitable choice of $L_{2}\left(I^{s}\right)$ kernels $\left\{K_{s}\right\}$ for the orthogonal system $\left\{g_{s}\right\}$ is that the Parseval's equality

holds.

$$
\|f\|^{2}=\sum_{s=0}^{\infty} s ! \int_{I^{s}}\left|K_{s}\left(\tau_{1}, \tau_{2}, \ldots, \tau_{s}\right)\right|^{2} d \tau_{1} d \tau_{2} \ldots d \tau_{s}
$$

Now we present a result for the space $L_{2}(\Omega, \mathscr{B}, \mu)$ which is similar in spirit to the celebrated Riesz-Fischer theorem for ordinary $L_{2}(\Sigma, \lambda)$ space with $\Sigma$ contained in a finite dimensional space like $R^{n}$ and $\lambda$ the Lebesgue measure.

Theorem 2.3: Given the orthogonal set $\left\{g_{s}\left(K_{s}, \cdot\right), s \in N_{+}\right\}$with $\left\{K_{s} \in L_{2}\left(I^{s}\right)\right\}$, a necessary and sufficient condition that there exists an $L_{2}\left(\Omega, \mathscr{B}_{B}, \mu\right)$ function with $\left\{K_{s}\right\}$ as it's Fourier-Wiener kernels is that the series

$$
\sum_{s=0}^{\infty} s ! \int_{I^{s}}\left|K_{s}\left(\tau_{1}, \tau_{2}, \ldots, \tau_{s}\right)\right|^{2} d \tau_{1} d \tau_{2} \ldots d \tau_{s}
$$

converges.

Proof: For detailed proof, see [1, Theorem 2].

Consider the vector space of all $\left\{K \equiv\left\{K_{s} \in \widehat{L}_{2}\left(I^{s}\right), s \in N_{+}\right\}\right\} \in \prod_{n=0}^{\infty} \widehat{L}_{2}\left(I^{n}\right) \equiv \mathscr{G}$. Let $G$ denote the completion of the vector subspace of $\mathscr{S}$ with respect to the scalar product

$$
\langle K, L\rangle \equiv \sum_{s=0}^{\infty} s ! \int_{I^{s}} K_{s}\left(\tau_{1}, \tau_{2}, \ldots, t_{s}\right) L_{s}^{*}\left(\tau_{1}, \tau_{2}, \ldots, \tau_{s}\right) d \tau_{1} d \tau_{2} \ldots d \tau_{s} .
$$

Clearly $G$ is a Hilbert space with the corresponding norm topology given by 


$$
\|K\|_{G} \equiv\left(\sum_{s=0}^{\infty} s ! \int_{I^{s}}\left|K_{s}\left(\tau_{1}, \tau_{2}, \ldots, \tau_{s}\right)\right|^{2} d \tau_{1} d \tau_{2} \ldots d \tau_{s}\right)^{1 / 2} .
$$

In view of Theorem 2.3 , we see that $L_{2}(\Omega, \mathscr{B}, \mu)$ is isometrically isomorphic to $G$. More specifically, define the map $\Phi: G \rightarrow L_{2}(\Omega, \mathfrak{B}, \mu)$ by

$$
\Phi(K) \equiv \sum_{s=0}^{\infty} g_{s}\left(K_{s}, \cdot\right)
$$

Clearly,

(1) $\Phi(\alpha K)=\alpha \Phi(K)$ for all scalars $\alpha$ and $K \in G$.

(2) $\Phi(K+L)=\Phi(K)+\Phi(L)$ for all $K, L \in G$.

(3) $\quad\|\Phi(K)\|_{L_{2}(\Omega, \mathscr{B}, \mu)}=\|K\|_{G}$.

Thus $\Phi$ is an isometric isomorphism of $G$ onto $L_{2}(\Omega, \mathscr{B}, \mu)$ and we express this by writing $G \Leftrightarrow L_{2}(\Omega, \mathscr{B}, \mu)$.

Set $\mathcal{G} \equiv L_{2}(\Omega, \mathfrak{B}, \mu)$ and define

$$
g_{n} \equiv\left\{g_{n}\left(K_{n}\right): \sqrt{n !} K_{n} \in \widehat{L}_{2}\left(I^{n}\right)\right\}
$$

This is the space of Wiener's homogeneous chaos. The celebrated Wiener-Ito decomposition follows as a corollary from the above discussions.

Corollary 2.4: $L_{2}(\Omega, \mathfrak{B}, \mu)=\mathfrak{g} \Leftrightarrow G$ and further, $\mathcal{G}=\sum_{n=0}^{\stackrel{\infty}{\oplus} \mathfrak{g}_{n}}$.

\section{Functional Analysis on $G$}

Here we consider some basic questions of analysis on the Hilbert space $L_{2}(\Omega, \mathfrak{B}, \mu)$ or equivalently $G$. Since $L_{2}(\Omega, \mathscr{B}, \mu)$ and $G$ are Hilbert spaces, bounded subsets $A \subset G$ and $\widehat{A} \subset L_{2}(\Omega, \mathscr{B}, \mu)$ are conditionally weakly compact. If closed then they are also weakly compact. The interesting question is what are the necessary and sufficient conditions for (strong) compactness. These questions were settled in a paper of the author in 1973 [2]. We present some of these results here.

Now for convenience, we shall replace $I$ by $R$ and extend the elements $\left\{K_{n}\right\}$ of $L_{2}\left(I^{n}\right)$ by setting $K_{n} \equiv 0$ outside the interval $I$. Let $\Pi_{n}$ denote the projection of $G$ to

$$
G_{n} \equiv\left\{K \in G: K=\left(K_{0}, K_{1}, \ldots, K_{n}, 0,0,0, \ldots\right)\right\} .
$$

Let $\Lambda_{h}, h \in R$, denote the translation operator:

$$
\Lambda_{h} K=\left(K_{0}, K_{1}(\cdot+h), K_{2}(\cdot+h, \cdot+h) \ldots K_{n}(\cdot+h, \cdot+, \ldots+h), \ldots\right) .
$$

Clearly $\Lambda_{h}: G \rightarrow G$.

Theorem 3.1: $A$ set $A \subset G$ is conditionally compact if, and only if,

(1) A is bounded,

(2) $\operatorname{Lim}_{h \rightarrow 0}\left\|\Lambda_{h} K-K\right\|_{G}=0$ uniformly with respect to $K \in A$,

(3) $\operatorname{Lim}_{n \rightarrow \infty}\left\|\left(1-\Pi_{n}\right) K\right\|_{G}=0$ uniformly with respect to $K \in A$.

Further, $A$ is compact if it is also closed. 
Proof: For detailed proof, see [2, Theorem 4].

Next we present a parallel result for the function space $L_{2}(\Omega, \mathscr{B}, \mu)$. Let $\left\{T_{t}, t \in R\right\}$ denote the ergodic group of measure preserving transformations of $\mathscr{B}$ into itself; and $P_{n}$ denote the projection of $L_{2}(\Omega, \mathscr{B}, \mu)$ to $H_{n}$ as defined below:

$$
H_{n} \equiv\left\{f \in L_{2}(\Omega, \mathscr{B}, \mu): f(w)=\sum_{s=0}^{n} g_{s}\left(K_{s}, w\right), K_{s} \in L_{2}\left(I^{s}\right), 0 \leq s \leq n\right\} .
$$

Theorem 3.2: $A$ set $F \subset L_{2}(\Omega, \Re, \mu)$ is conditionally compact if, and only if,

$$
F \text { is bounded }
$$

$$
\begin{aligned}
& \operatorname{Lim}_{t \rightarrow 0} \int_{\Omega}\left|f\left(T_{t} w\right)-f(w)\right|^{2} d \mu(w)=0 \text { uniformly with respect to } f \in F, \\
& \operatorname{Lim}_{n \rightarrow \infty} \int_{\Omega}\left|\left(1-P_{n}\right) f(w)\right|^{2} d \mu(w)=0 \text { uniformly with respect to } f \in F .
\end{aligned}
$$

Proof: The proof follows from Theorem 2.4 and the isomorphism $\Phi$ (see [2, Corollary 2]).

Before we conclude this section, we would like to present another interesting fact which is particularly important in applications. So far we have considered standard Wiener process, that is $\&(w(t) w(s))=t \wedge s$. This means that the corresponding white noise has power spectral density 1. For arbitrary power density we may consider $\mathscr{E}(w(t) w(s))=(1 / 2 \lambda) t \wedge s$, for $\lambda>0$. The smaller the $\lambda$, the larger is the noise power. Note that this is also equivalent to time scaling of the standard Brownian motion. Let $\mu_{\lambda}$ denote the corresponding Wiener measure and accordingly replace $L_{2}(\Omega, \mathfrak{B}, \mu)$ by $L_{2}\left(\Omega, \mathfrak{B}, \mu_{\lambda}\right)$. In this case the orthogonal set $\left\{g_{n}\right\}$ has the following basic properties:

(Q1) Same as $(P 1)$ with $\mu$ replaced by $\mu_{\lambda}$.

$(Q 2)$ Same as $(P 2)$ with $\mu$ replaced by $\mu_{\lambda}$.

(Q3) For each $K_{n} \in \widehat{L}_{2}\left(I^{n}\right)$ and $L_{n} \in \widehat{L}_{2}\left(I^{n}\right)$

$$
\begin{aligned}
& \left\langle g_{n}\left(K_{n}, \cdot\right), g_{n}\left(L_{n}, \cdot\right)\right\rangle_{\mu_{\lambda}} \equiv \int_{\Omega} g_{n}\left(K_{n}, w\right) g_{n}\left(L_{n}, w\right) d \mu_{\lambda}(w) \\
= & \left(n ! /(2 \lambda)^{n}\right) \int_{I^{n}} K_{n}\left(\tau_{1}, \tau_{2}, \ldots, \tau_{n}\right) L_{n}^{*}\left(\tau_{1}, \tau_{2}, \ldots, \tau_{n}\right) d \tau_{1} d \tau_{2} \ldots d \tau_{n} .
\end{aligned}
$$

(Q4) Same as (P4) with $\mu$ replaced by $\mu_{\lambda}$.

With this modification $G$ is replaced by $G_{\lambda}$ which is given the natural norm topology

$$
\|K\|_{\lambda} \equiv\left(\sum_{n=0}^{\infty}\left(n ! /(2 \lambda)^{n}\right) \int_{I^{n}}\left|K_{n}\left(\tau_{1}, \tau_{2}, \ldots, \tau_{n}\right)\right|^{2} d \tau_{1} d \tau_{2} \ldots d \tau_{n}\right)^{1 / 2}
$$

All the results presented in this section remain valid for the spaces $L_{2}\left(\Omega, \mathscr{B}, \mu_{\lambda}\right)$ and $G_{\lambda}$ which are, of course, isometrically isomorphic. Note that for $\lambda=1 / 2$, we have $G_{1 / 2}=G$.

Theorem 3.3: The system of Hilbert spaces $\left\{G_{\lambda}, \lambda>0\right\}$ forms a totally ordered Lattice with $G_{\lambda} \subset G_{\beta}$ for $0<\lambda<\beta$, and by virtue of the isomorphism, $L_{2}\left(\Omega, \Re, \mu_{\lambda}\right) \subset L_{2}\left(\Omega, \Re, \mu_{\beta}\right)$ and the injection is continuous.

So far we have presented some results due to the author that appeared in the mid-sixties and early seventies. 'These are regular functionals of white noise. 


\section{Generalized Functionals of the First Kind}

In this section we construct a class of mild generalized functionals, which we call generalized functionals of the first kind, strongly related to regular functionals as discussed in Section 2. Let $H$ denote the vector space

$$
H \equiv\left\{K \in \mathscr{G}:\|K\|_{H} \equiv\left(\sum_{n=0}^{\infty}\left\|K_{n}\right\|_{L_{2}\left(I^{n}\right)}^{2}\right)^{1 / 2}<\infty\right\} .
$$

We assume that $H$ has been completed with respect to the scalar product

$$
(K, L) \equiv \sum_{n=0}^{\infty}\left(K_{n}, L_{n}\right)_{L_{2}\left(I^{n}\right)},
$$

so that it becomes a real Hilbert space. It is easy to verify that for each $L \in G$, we have

$$
\|L\|_{H} \leq\|L\|_{G}
$$

In other words, $G \subset H$ but the reverse inclusion is not true and the injection $G \rightarrow H$ is continuous. Let $L$ be any element of $H$ and define the linear functional on $G$ by setting $\ell_{L}(K)=(L, K)_{H}$. Clearly

$$
\left|\ell_{L}(K)\right| \leq\|L\|_{H}\|K\|_{H} \leq\|L\|_{H}\|K\|_{G}
$$

Thus every $L \in H$ determines a continuous linear functional on $G$. In fact, the space of continuous linear functionals on $G$ is much larger. For $L \in \mathscr{K}$ and $K \in G$, we have

$$
\begin{gathered}
\langle L, K\rangle=\sum_{n=0}^{\infty}\left(L_{n}, K_{n}\right)_{L_{2}\left(I^{n}\right)}=\sum_{n=0}^{\infty}\left((1 / \sqrt{n !}) L_{n},(\sqrt{n !}) K_{n}\right)_{L_{2}\left(I^{n}\right)} \\
\leq\left(\sum_{n=0}^{\infty}(1 / n !)\left\|L_{n}\right\|_{L_{2}\left(I^{n}\right)}^{2}\right)^{1 / 2}\left(\sum_{n=0}^{\infty}(n !)\left\|K_{n}\right\|_{L_{2}\left(I^{n}\right)}^{2}\right)^{1 / 2} \\
\leq\left(\sum_{n=0}^{\infty}(1 / n !)\left\|L_{n}\right\|_{L_{2}\left(I^{n}\right)}^{2}\right)^{1 / 2}\|K\|_{G} .
\end{gathered}
$$

It is clear from this inequality that for each $K \in G$, the expression on the right-hand side is finite if, and only if, $L \in 96$ is such that

$$
\sum_{n=0}^{\infty}(1 / n !)\left\|L_{n}\right\|_{L_{2}\left(I^{n}\right)}^{2}<\infty
$$

Identify $H$ with its dual $H^{*}$. Let $G^{*}$ denote the dual of $G$. The inequality (4.2) characterizes the dual $G^{*}$, that is, the continuous linear functionals on $G$ are given by only those elements of $\mathscr{T}$ which satisfy (4.3). Thus the pairing on the left of (4.2) can be interpreted as $G^{*}, G$ pairing giving

$$
\left|\langle L, K\rangle_{G^{*}, G}\right| \leq\|L\|_{G^{*}}\|K\|_{G} .
$$

Then $G^{*}$ is also a Hilbert space with the norm topology

$$
\|L\|_{G^{*}} \equiv\left(\sum_{n=0}^{\infty}(1 / n !)\left\|L_{n}\right\|_{L^{2}\left(I^{n}\right)}^{2}\right)^{1 / 2} .
$$

It is easy to show that $G$ is dense in $H$ and $H$ is dense in $G^{*}$. Thus we have proved the 
following result.

Lemma 4.1: Consider the triple $G, H, G^{*}$ as defined above. They are all Hilbert spaces and the embeddings $G \rightarrow H \rightarrow G^{*}$ are continuous and dense.

Using this result and the isomorphism $\Phi$ as defined in Section 2, we can construct a class of generalized functionals as follows. Since $G$ is dense in $H$ which, in turn, is dense in $G^{*}$, by the principle of extension by continuity, we can extend $\Phi$ all the way up to $G^{*}$ in such a way that it's restriction to $G$ is $\Phi$ itself. We shall denote the extension also by $\Phi$ itself. We define

$$
\mathcal{G} \equiv \Phi(G), \mathfrak{H} \equiv \Phi(H), \mathcal{G}^{*} \equiv \Phi\left(G^{*}\right)
$$

Hence we have the following result.

Theorem 4.2: The class $\mathrm{G}$ is the space of regular $L_{2}$ functionals of Brownian motion while the two classes 16 and $\mathrm{g}^{*}$ are generalized functionals of Brownian motion with $\mathrm{g}$ being their test functionals and they satisfy the following diagram:

$$
\begin{aligned}
& G \rightarrow H \rightarrow G^{*} \\
& \mathcal{G} \rightarrow \mathfrak{H} \rightarrow \mathcal{G}^{*} .
\end{aligned}
$$

The action of an element of $\mathcal{g}^{*}$ on those of the regular Brownian functionals $\mathcal{G}=L_{2}(\Omega, \mathfrak{B}, \mu)$ is given by the duality pairing

and clearly

$$
\left\langle f^{*}, f\right\rangle_{\mathcal{G}^{*}, \mathcal{G}}=\sum_{n=0}^{\infty}(1 / n !)\left(g_{n}\left(L_{n}\right), g_{n}\left(K_{n}\right)\right)_{L_{2}(\Omega, \mathfrak{B}, \mu}=\langle L, K\rangle_{G^{*}, G}
$$

$$
\left|\left\langle f^{*}, f\right\rangle_{\mathcal{G}^{*}, \mathfrak{g}}\right| \leq\|L\|_{G^{*}}\|K\|_{G}
$$

Note that for every $f \in \mathcal{G}$ there exists an $f^{*} \in \mathcal{G}^{*}$ such that

$$
\left\langle f^{*}, f\right\rangle=\|f\|_{g}
$$

For example, if $f \leftrightarrow K$, then $f^{*}$ given by $f^{*} \equiv \Phi(L)$ for $L \equiv\left(L_{0}, L_{1}, L_{3}, \ldots, L_{n}, \ldots\right)$ with $L_{n}=\left(n ! /\|K\|_{G}\right) K_{n}$ has the required property and, further, $\left\|f^{*}\right\|_{\mathrm{G}^{*}}=1$.

Remark 4.3: Even though the results in this section are stated for one dimensional Brownian motion, they are also valid for multidimensional case. This requires only use of symmetric multilinear forms for the multiple Wiener integrals. Further, the results also apply to multiparamter Brownian motion.

\section{Generalized Functionals of White Noise II}

It is known that the derivative of the Wiener process does not exist in the classical sense but in the sense of distribution it has derivatives of all orders. Let $\mathscr{D}$ denote the space of $C^{\infty}$ functions on $I$ with compact support and $\mathscr{T}^{*}$ the space of Schwarz distributions on $I$. Then we can consider the white noise or the derivative of the Wiener process $\dot{w}$ as a random distribution in the sense that its action on $\mathscr{D}$ is given by

$$
\dot{w}(\phi)=(-1) \int_{I} w(t) \dot{\phi}(t) d t \text { for all } \phi \in \mathscr{D} .
$$


Note that $\dot{w}(\phi)$ is a well defined Gaussian random variable, parametrized by $\phi \in \mathscr{T}$, with mean zero and covariance $\|\phi\|^{2} \equiv \int_{I}|\phi(t)|^{2} d t$. In fact the $n$th derivative of $w$, denoted by $w^{(n)}$, is also a random distribution and

$$
w^{(n)}(\phi)=(-1)^{n} \int_{I} w(t) \phi^{(n)}(t) d t \text { for all } \phi \in \mathscr{D} .
$$

Clearly this is also a Gaussian random variable, parametrized by $\phi \in \mathscr{D}$, with mean zero and variance $\int\left|\phi^{(n-1)}(t)\right|^{2} d t$. Thus the derivatives of the Wiener process are actually stochastic distributions in the sense of Schwarz and we may call them generalized random processes. It is easy to see that for any fixed $s \in(0, T), f_{s}(w) \equiv \dot{w}(s)$ is an unbounded linear functional of Brownian motion and is not an element of $L_{2}(\Omega, \mathfrak{B}, \mu)$, and hence it is not a regular functional. Similarly, $F_{s}(w) \equiv(\dot{w}(s))^{n}$ is a homogeneous functional of white noise of degree $n$ but it is not an element of $L_{2}(\Omega, \mathscr{B}, \mu)$. This certainly justifies the need of generalized functionals and we shall see later that the functionals mentioned above are well defined generalized functionals.

Note that the characteristic function of the white noise is given by

$$
\&\{\exp (i \dot{w}(\phi))\} \equiv \exp -(1 / 2) \int_{I}|\phi(t)|^{2} d t \equiv C(\phi) .
$$

It is clear that the function $C$ is also well defined on the Hilbert space $\Pi \equiv L_{2}(I)$, that is, it is continuous, positive definite, and $C(0)=1$. However, there exists no countably additive measure $\mu$ on $\mathfrak{B}(\Pi)$ that satisfies the relation

$$
C(\phi)=\int_{H} e^{i(x, \phi)} d \mu(x), \phi \in \Pi .
$$

A necessary and sufficient condition for countable additivity is provided by Minlos-Sazanov theorem (generalizing Bochner's theorem) which states that the measure is countably additive if, and only if, the corresponding covariance operator in Nuclear.

Thus, if we consider the functional $C$ on the Sobolev space $H_{0}^{1}$ then the white noise has a countably additive measure on the dual $\left(H_{0}^{1}\right)^{*}=H^{-1}$. In this case

$$
C(\phi) \equiv \int_{H^{-1}} \exp (i\langle x, \phi\rangle) d \mu(x) .
$$

We can justify this as follows. It is easy to verify that

$$
\&(\langle\dot{w}, \phi\rangle)^{2}=\int_{I \times I}(t \wedge \tau) \dot{\phi}(t) \dot{\phi}(\tau) d t d \tau \equiv\langle R \phi, \phi\rangle .
$$

Here we have used $\langle$,$\rangle to denote the duality pairing between H^{-1}$ and $H_{0}^{1}$. It is clear that the kernel $K(t, \tau) \equiv t \wedge \tau$ is symmetric and positive. Since $\int K(t, t)=T^{2} / 2<\infty$, the

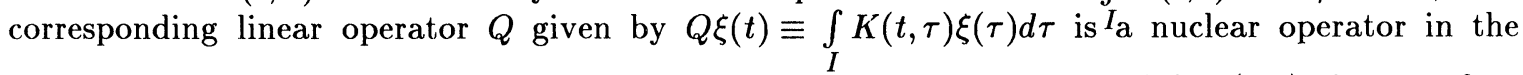
Hilbert space $\Pi=L_{2}(I)$. Hence the covariance operator $R$, as defined by (5.6), is a nuclear operator from $H_{0}^{1}$ to $H^{-1}$. Similarly one can verify that the $m$ th distributional derivative $w^{(m)}$ of the Wiener process $w$ induces a countably additive Gaussian measure on $H^{-m}$ which is the dual of $H_{0}^{m}$. Here we are interested only in the white noise since the Wiener's multiple integrals are functionals of the white noise. 
Thus to catch the support of the white noise measure, we must choose a larger space, larger than II, for example the dual $S^{*}$ of a nuclear space $S \subseteq H_{0}^{1}$ so that

$$
\mathfrak{D} \rightarrow S \rightarrow \Pi \rightarrow S^{*} \rightarrow \mathscr{D}^{*} .
$$

Let $\left(S^{*}, \mathfrak{B}\left(S^{*}\right), \mu\right)$ denote the measure space with $\mu$ denoting the white noise measure on $\mathfrak{B}\left(S^{*}\right)$ and let $M\left(S^{*}, \mathscr{B}\left(S^{*}\right), \mu\right) \equiv M\left(S^{*}, \mu\right)$ denote the class of $\mu$-measurable functions on $S^{*}$ and let $L_{2}\left(S^{*}, \mu\right)$ denote the Hilbert space of $\mu$-measurable and square-integrable functions on $S^{*}$. These are measurable functionals on $S^{*}$.

We shall use $F\left(S^{*}, \mu\right)$ to denote the universal space of generalized functionals of white noise. That is, these functionals are not necessarily $\mathfrak{B}\left(S^{*}\right)$ measurable, nor are they defined pointwise. They are generally distributions and are characterized by their actions on suitable test functionals.

Thus the multiple Wiener-Ito integrals can also be written as

$$
g_{n}(\sigma)=\int_{I^{n}} K_{n}\left(\tau_{1}, \tau_{2}, \ldots, \tau_{n}\right) \sigma\left(\tau_{1}\right) \sigma\left(\tau_{2}\right) \ldots \sigma\left(\tau_{n}\right) d \tau_{1} d \tau_{2} \ldots d \tau_{n}, \sigma \in S^{*}
$$

In other words, these are the multiple white noise integrals considered as measurable functions on $S^{*}$. As stated in the introduction, Hida constructed his generalized functionals by introducing a Fourier-like transform on the space $L_{2}\left(S^{*}, \mu\right)$ as follows

$$
\left(\Im_{f}\right)(\xi) \equiv \int_{S^{*}} \exp (i\langle x, \xi\rangle) f(x) d \mu(x), \xi \in S,
$$

where the duality pairing in (5.8) is between $S^{*}$ and $S$.

For convenience we shall denote this by

$$
\widehat{f}(\xi) \equiv(\mathscr{F} f)(\xi) .
$$

It is clear from this definition that for $f \in L_{2}\left(S^{*}, \mu\right), \widehat{f}(\xi)$ is a bounded analytic function on $S$. Indeed for $f=g_{n}$, it is not difficult to verify that

$$
\widehat{g}_{n}(\xi)=(i)^{n}\left\{\exp -(1 / 2)\|\xi\|^{2}\right\} \int_{I^{n}} K_{n}\left(\tau_{1}, \tau_{2}, \ldots, \tau_{n}\right) \xi\left(\tau_{1}\right) \xi\left(\tau_{2}\right) \ldots \xi\left(\tau_{n}\right) d \tau_{1} d \tau_{2} \ldots d \tau_{n}:
$$

where $\|\xi\|$ is the $L_{2}(I)$ norm of $\xi$. The proof is easily obtained for simple functions $K_{n}$ by keeping in mind the fact that the increments of Brownian motion on disjoint intervals are independent Gaussian random variables with mean zero and variance equal to the lengths of the intervals. For general $K_{n} \in L_{2}\left(I^{n}\right)$, one then uses the density argument and the fact that simple functions are dense in $L_{2}$. Defining $C(\xi) \equiv \exp (-1 / 2)\|\xi\|^{2}$, it follows from (5.9) that

$$
\widehat{g}_{n}(\xi) \equiv\left(\mathscr{F}_{n}\right)(\xi)=g_{n}(i \xi) C(\xi) \text {. }
$$

Thus for $\phi(x) \equiv \sum_{n=0}^{\infty} g_{n}(x)$, we have

$$
\widehat{\phi}(\xi)=C(\xi) \sum_{n=0}^{\infty} g_{n}(i \xi) .
$$

It is known that the standard Fourier transform is an isometric isomorphism of $L_{2}$ spaces over finite dimensional spaces. Since $S^{*}$ is different from $S$ and also they are infinite dimensional, the 
Fourier-Hida transform is not an isometry. However, it follows from the above relations that it is an isomorphism. Indeed if $\widehat{g}_{n}(\xi)=0$ for all $\xi \in S$ then $g_{n}(i \xi)=0$ for all $\xi \in S$ and hence $K_{n} \equiv 0$. Thus, for any $\phi \in L_{2}\left(S^{*}, \mu\right), \widehat{\phi}(\xi)=0$ for all $\xi \in S$ implies that $\phi=0$ or equivalently $K(\in G)=0$.

Now we are prepared to introduce the generalized functionals constructed by Hida [6-8]. In this section we take $I^{n}=R^{n}$. Define

$$
\mathfrak{H}_{n} \equiv\left\{g_{n}\left(K_{n}\right) \equiv g_{n}\left(K_{n}, \cdot\right), K_{n} \in \widehat{L}_{2}\left(I^{n}\right)\right\}
$$

where $g_{n} \in L_{2}\left(S^{*}, \mu\right)$ is a homogeneous functional of white noise of degree $n$ as defined by the expression (5.7). Then define

$$
\mathscr{F}_{n} \equiv \mathscr{F}\left(\mathcal{H}_{n}\right)
$$

This is a linear vector space isomorphic to $\mathfrak{H}_{n}$. For any real number $m \geq 0$, let

$$
H^{m} \equiv\left\{\phi \in \widehat{L}_{2}\left(R^{n}\right): D^{\alpha} \phi \in L_{2}\left(R^{n}\right),|\alpha| \leq m\right\}
$$

denote the Sobolev space of real valued symmetric functions on $R^{n}$ with the usual norm topology

$$
\|\phi\|_{H^{m}} \equiv\left(\sum_{|\alpha| \leq m}\left\|D^{\alpha} \phi\right\|_{L_{2}\left(R^{n}\right)}^{2}\right)^{1 / 2}
$$

Now for $m=m(n) \equiv((n+1) / 2)$, it is known from the Sobolev embedding theorem that the injection $H^{m(n)} \rightarrow C\left(R^{n}\right)$ is continuous. This is the correct number for construction of generalized functionals as seen below. Since the dual of $H^{m(n)}$ is $H^{-m(n)}$ it is clear that

$$
H^{m(n)} \rightarrow \widehat{L}_{2}\left(R^{n}\right) \rightarrow H^{-m(n)}
$$

where the injections are continuous and dense. Define

$$
\begin{aligned}
& \mathcal{H}_{n}^{m(n)} \equiv\left\{g_{n}\left(K_{n}, \cdot\right) \in L_{2}\left(S^{*}, \mu\right): K_{n} \in H^{m(n)}\right\}, \\
& \mathscr{F}_{n}^{m(n)}=\mathscr{F}\left(\mathcal{J G}_{n}^{m(n)}\right)
\end{aligned}
$$

and

$$
\mathscr{F}_{n} \equiv \mathscr{F}\left(\mathfrak{H}_{n}\right)
$$

where $\mathfrak{H}_{n}$ is given by (5.12). Identifying $\mathscr{F}_{n}$ with its topological dual $\mathscr{F}_{n}^{*}$, it follows from (5.14) that

$$
\mathscr{F}_{n}^{m(n)} \rightarrow \mathcal{F}_{n} \rightarrow \mathcal{F}_{n}^{-m(n)}
$$

Theorem 5.1 (Hida): Under the above assumptions, the following diagram holds:

$$
\begin{gathered}
\mathscr{F}_{n}^{m(n)} \rightarrow \mathcal{F}_{n} \rightarrow \mathscr{F}_{n}^{-m(n)} \\
\cong \cong \\
\mathfrak{F}_{n}^{m(n)} \rightarrow \mathfrak{F}_{n} \rightarrow \mathcal{F}_{n}^{-m(n)} .
\end{gathered}
$$


Proof: The Fourier-Hida transform is an isomorphism. Its inverse is well defined on both $\mathscr{F}_{n}^{m(n)}$ and $\mathscr{F}_{n}$. Since the embeddings in (5.14) are dense, again by the principle of extension by continuity, the inverse of the Fourier-Hida transform can be extended to $\mathcal{F}_{n}^{-m(n)}$. Thus by application of the inverse transform, one obtains the last column of (5.17) giving the result as indicated by the diagram.

Recall that $\mathcal{H}_{n}$ is the space of regular functionals of degree $n$ of the white noise $\left(S^{*}, \mu\right)$. The elements of $\mathfrak{G}_{n}^{-m(n)}$ are generalized functionals of white noise.

For example, consider the functional

$$
\widehat{\Psi}(\xi) \equiv \int_{R^{s}} K_{s}\left(\vartheta_{1}, \vartheta_{2}, \ldots, \vartheta_{s}\right)\left(\xi\left(\vartheta_{1}\right)\right)^{n_{1}}\left(\xi\left(\vartheta_{2}\right)\right)^{n_{2}} \ldots\left(\xi\left(\vartheta_{s}\right)\right)^{n} s_{d} d \vartheta_{1} d \vartheta_{2} \ldots d \vartheta_{s}
$$

where $\sum_{i=1}^{s} n_{i}=n$. Clearly $\widehat{\Psi}$ is a homogeneous functional of degree $n$ on $S$ but it is not an element of $\mathcal{F}_{n}$. It is however, an element of $\mathcal{F}_{n}^{-m(n)}$. Hence by the Fourier-Hida inverse transform $\Psi \equiv \mathscr{F}^{-1}\left((i)^{n} C \widehat{\Psi}\right) \in \mathcal{F}_{n}^{-m(n)}$, where $C$ is the characteristic function of white noise as introduced earlier. Hence the functionals in the class $\mathfrak{H}_{n}^{-m(n)}$ have the same integral form as (5.18) with $\xi \in S$ replaced by $x \in S^{*}$ defining the generalized functional of white noise.

Now we wish to extend the above result to infinitely many variables. Recall that the embedding $H^{m(n)} \rightarrow L_{2}\left(R^{n}\right)$ is continuous and the embedding constant is $\leq 1$. We introduce a norm topology on $\mathbb{H}^{+} \equiv \sum_{n=0} \underset{\oplus}{\oplus} \mathcal{G}_{n}^{m(n)}$ as follows: For $\phi \in \mathbb{H}^{+}$we write

$$
\|\phi\|_{\mathbb{H}^{+}}^{2} \equiv \sum_{n=0}^{\infty} n !\left\|K_{n}\right\|_{H}^{2} m m(n) \text {. }
$$

Similarly for $\mathbb{H} \equiv \sum \underset{n=0}{\oplus_{0}} \mathfrak{H}_{n}$ we have already the norm topology induced by

$$
\|\phi\|_{\mathbb{H}}^{2} \equiv \sum_{n=0}^{\infty} n !\left\|K_{n}\right\|_{L_{2}\left(R^{n}\right)}^{2}
$$

Letting $\mathbb{H}^{-}$denote the dual of $\mathbb{H}^{+}$we have the following result.

Theorem 5.2: Under the assumptions of Theorem 5.1, and the norm topologies as introduced above for the spaces $\mathbb{H}^{+}, \mathbb{H}$, and $\mathbb{H}^{-}$and identifying $\mathbb{H}$ with its own dual, we have the following embeddings

$$
\mathbb{H}^{+} \rightarrow \mathbb{H}_{\rightarrow} \mathbb{H}^{-}
$$

which are continuous and dense.

Define

$$
\mathcal{F}^{+} \equiv \sum \bigoplus_{n=0}^{\infty} \mathcal{F}_{n}^{m(n)}, \mathcal{F} \equiv \sum \bigoplus_{n=0}^{\infty} \mathcal{F}_{n}, \mathcal{F}^{-} \equiv \sum_{n=0}^{\infty} \bigoplus_{n}^{-m(n)}
$$

Then it follows from Fourier-Hida transform and Theorem 5.2 that the following result holds.

Corollary 5.3:

$$
\begin{gathered}
\mathscr{F}^{+} \rightarrow \mathscr{F}^{\rightarrow} \rightarrow \mathcal{F}^{-} \\
\cong \cong \\
\mathbb{H}^{+} \rightarrow \mathbb{H}^{\rightarrow} \rightarrow \mathbb{H}^{-} .
\end{gathered}
$$


In the following two sections we present a much more wider class of generalized functionals of white noise as introduced by the author [5]. These functionals cover the Hida class and much more and applies to problems where other classes fail. First we shall introduce the class of generalized functionals based on $L_{p}$ spaces and then those based on Sobolev spaces $\Psi^{p, m}$.

\section{6. $L_{p}$-Based Generalized Functionals of White Noise III}

For each $p \in[1, \infty]$, and each positive integer $n$, let $\widehat{L}_{p}\left(I^{n}\right)$ denote the equivalence classes of symmetric functions from the class $L_{p}\left(I^{n}\right)$ where the Lebesgue measure $\ell(I)$ of $I$ is finite. Define

$$
G^{p, n} \equiv\left\{K_{n} \in \widehat{L}_{p}\left(I^{n}\right) \text { with the norm }\left\|K_{n}\right\|_{G} p, n \equiv(n !)^{1 / p}\left\|K_{n}\right\|_{L_{p}\left(I^{n}\right)}<\infty\right\} .
$$

Clearly, $G^{p, n}$ is a Banach space. For each $L_{n} \in G^{p, n}$, define the functional

$$
\phi_{n}\left(L_{n}\right) \equiv \int_{I^{n}} L_{n}\left(\tau_{1}, \tau_{2}, \ldots, \tau_{n}\right) \dot{w}\left(\tau_{1}\right) \dot{w}\left(\tau_{2}\right) \ldots \dot{w}\left(\tau_{n}\right) d \tau_{1} d \tau_{2} \ldots d \tau_{n}
$$

where $\dot{w} \in S^{*}$ with the white noise measure $\mu$. We consider this functional as a $\mu$ measurable function on $S^{*}$. We shall use $M\left(S^{*}, \mu\right)$ to denote all $\mu$ measurable functions on $S^{*}$. Clearly, for each $p \geq 2$ and $K_{n} \in G^{p, n}, z \equiv \phi_{n}\left(K_{n}\right)$ defines a regular homogeneous chaos of degree $n$ of Wiener-Ito class and belongs to $M\left(S^{*}, \mu\right)$. Define

$$
\mathrm{g}^{p, n} \equiv\left\{z \in M\left(S^{*}, \mu\right): z=\phi_{n}\left(K_{n}\right), K_{n} \in G^{p, n}\right\} .
$$

This is a linear vector space. For $z \in \mathfrak{G}^{p, n}$, corresponding to the kernel $K_{n} \in G^{p, n}$, define

$$
\|z\|_{g^{p, n}} \equiv\left\|K_{n}\right\|_{G} p, n \text {. }
$$

Lemma 6.1: For $2 \leq p<\infty, \mathrm{g}^{p, n}$ is a Banach space with respect to the norm topology given by (6.3) and it is isometrically isomorphic to $G^{p, n}$.

Proof: See [5, Lemma 3.1].

The functionals belonging to the class $\mathrm{g}^{p, n}, p \geq 2$ are regular functionals defined in the Wiener-Ito sense. For $1 \leq q<2$ and $L_{n} \in G^{q, n}$, the random element $z^{\prime} \equiv \phi_{n}\left(L_{n}\right)$ is not defined in the classical Wiener-Ito sense. However, we can define them in a weak sense as generalized random elements as given in [5].

Recall that $F\left(S^{*}, \mu\right)$ denotes the (universal) space of generalized functionals of the white noise $\left(S^{*}, \mu\right)$, generally not defined pointwise.

Lemma 6.2: For each $L_{n} \in G^{q, n}, 1 \leq q \leq 2$, the functional $z^{*} \equiv \phi_{n}\left(L_{n}\right)$ is a well defined random element on $\left(S^{*}, \mu\right)$ in the sense that it is an element of $F\left(S^{*}, \mu\right)$ and that it is a continuous linear functional on $\mathrm{g}^{p, n}$.

Proof: For detailed proof see [5, Lemma 3.2, pp. 143]. Due to its fundamental importance, we will present an outline of the proof. Since $L_{2}\left(I^{n}\right)$ is dense in $L_{q}\left(I^{n}\right), 1 \leq q \leq 2$, there exists a sequence $\left\{L_{n, s}, s=1,2,3, \ldots\right\} \in L_{2}\left(I^{n}\right)$ such that $L_{n, s} \stackrel{s}{\rightarrow} L_{n}$ (strongly) in $L_{q}\left(I^{n}\right)$. Then the sequence $\left\{z_{s}^{*} \equiv \phi_{n}\left(L_{n, s}\right), s=1,2, \ldots\right\}$ is a sequence of regular functionals. Hence, for any $z \in \mathcal{G}^{p, n}$, with $p^{-1}+q^{-1}=1$, which, by the previous lemma, has the representation $z=\phi_{n}\left(K_{n}\right)$ for some $K_{n} \in G^{p, n}$, we have 


$$
\begin{gathered}
\&\left(z_{s}^{*}, z\right)=n !\left(L_{n, s}, K_{n}\right)_{L_{2}\left(I^{n}\right), L_{2}\left(I^{n}\right)} \\
=n !\left(L_{n, s}, K_{n}\right)_{L_{q}\left(I^{n}\right), L_{p}\left(I^{n}\right)} \\
=\left(L_{n, s}, K_{n}\right)_{G^{q, n}, G} p, n .
\end{gathered}
$$

Since $L_{n, s} \stackrel{s}{\rightarrow} L_{n}$ in $L_{q}\left(I^{n}\right)$, it also converges weakly to the same element. Thus the limit of the right-hand expression exists for all $K_{n} \in G^{p, n}$. Hence the limit of the expression on the lefthand side is well defined for every $z \in \mathfrak{g}^{p, n}$. We call the limit of $z_{s}^{*}$, in the above sense, the generalized random variable $z^{*}$ given by $z^{*} \equiv \phi_{n}\left(L_{n}\right)$. Hence $z^{*}$ is well defined as a continuous linear functional on $\mathrm{g}^{p, n}$, and, therefore, it belongs to $F\left(S^{*}, \mu\right)$.

On the basis of the above result we can introduce a duality pairing between the spaces $\mathrm{g}^{q, n}$ and $\mathrm{g}^{p, n}$ with $1 \leq q \leq 2 \leq p<\infty$ and $p^{-1}+q^{-1}=1$ as follows:

$$
\left\langle z^{*}, z\right\rangle_{\mathfrak{G}^{q, n}, \mathfrak{g}^{p, n}} \equiv \mathcal{E}\left(z^{*}, z\right)=\left(L_{n}, K_{n}\right)_{G^{q, n}, G}^{p, n} .
$$

where $z^{*} \equiv \phi_{n}\left(L_{n}\right), L_{n} \in G^{q, n}$ and $z \equiv \phi_{n}\left(K_{n}\right), K_{n} \in G^{p, n}$.

We introduce a norm topology on $\mathrm{g}^{q, n}$ by setting

$$
\left\|z^{*}\right\|_{\mathrm{g}^{q, n}} \equiv \operatorname{Sup}\left\{\left|\left\langle z^{*}, z\right\rangle\right|,\|z\|_{\left.\mathrm{g}^{p, n}=1\right\}} .\right.
$$

Now we can present the following fundamental result.

Theorem 6.3: For each nonnegative integer $n$, and $1 \leq q \leq 2 \leq p<\infty$ with $p$ being conjugate of $q$ we have

$$
\begin{gathered}
\mathrm{g}^{p, n} \rightarrow \mathrm{g}^{2, n} \rightarrow \mathrm{g}^{q, n}=\left(\mathcal{G}^{p, n}\right)^{*} ; \\
G^{p, n} \rightarrow G^{2, n} \rightarrow G^{q, n} \\
\mathrm{~g}^{p, n} \rightarrow \mathrm{g}^{2, n} \rightarrow \mathrm{g}^{q, n} ;
\end{gathered}
$$

where all the injections are continuous and dense.

Proof: For detailed proof, see [5, Theorem 3.1].

We note that, for $1 \leq q<2, \mathrm{~g}^{q, n}$ is a system of generalized functionals of the white noise $\left(S^{*}, \mu\right)$. This system is more general than $\mathrm{g}^{2, n}$.

Remark 6.4: The fact that $z^{*} \in \mathrm{G}^{q, n}$ does not in any sense, imply that $\mathscr{E}\left|z^{*}\right|^{q}<\infty$, that is, $z^{*} \notin L_{q}\left(S^{*}, \mu\right)$.

Now we consider nonhomogeneous generalized functionals of degree $N \in N_{+}$. Define, for $r \geq 1, N<\infty$, the Banach space

$$
G_{r, N} \equiv\left\{L=\left(L_{0}, L_{1}, L_{2}, \ldots, L_{N}\right): L_{n} \in G^{r, n}, 0 \leq n \leq N\right\}
$$

with the norm topology

$$
\|L\|_{G_{r, N}} \equiv\left(\sum_{n=0}^{N} n !\left\|L_{n}\right\|_{L_{r}\left(I^{n}\right)}^{r}\right)^{1 / r} .
$$

Since $\ell(I)<\infty$, it is easy to verify that, for $1 \leq q \leq 2 \leq p<\infty, G_{p, N} \rightarrow G_{q, N}$ and that there exists a positive constant $C_{p, N}$ such that for each $L \in G_{p, N}$ we have 


$$
\|L\|_{G_{q, N}} \leq C_{p, N}\|L\|_{G_{p, N}}
$$

for every finite positive integer $N$. The injection is continuous and dense. The upper bound of the embedding constant can be shown to be

$$
C_{p, N} \leq\left(\sum_{n=0}^{N} n !(\ell(I))^{n}\right)^{(p-2) / p} .
$$

Now, we introduce the map $\Phi_{N}$ as

$$
\Phi_{N}(K) \equiv \sum_{n=0}^{N} \int_{I^{n}} K_{n}\left(\tau_{1}, \tau_{2}, \ldots, \tau_{n}\right) \dot{w}\left(\tau_{1}\right) \dot{w}\left(\tau_{2}\right) \ldots \dot{w}\left(\tau_{n}\right) d \tau_{1} d \tau_{2} \ldots d \tau_{n}
$$

defined for any $K \in G_{r, N}, r \geq 1$. For any $r \geq 1$ this defines a larger class of functionals on $\left(S^{*}, \mu\right)$ given by

$$
\mathfrak{G}_{r, N} \equiv\left\{z \in F\left(S^{*}, \mu\right): z=\Phi_{N}(K), \text { for some } K \in G_{r, N}\right\}
$$

As before, we can introduce a norm topology on $\mathfrak{G}_{r, N}$ by defining

$$
\|z\|_{\mathcal{G}_{r, N}} \equiv\|K\|_{G_{r, N}} \text {, for } z=\Phi_{N}(K), K \in G_{r, N} \text {. }
$$

This makes $\Phi_{N}$ an isometric isomorphism of $G_{r, N}$ onto $\mathfrak{G}_{r, N}$. This is stated more precisely in the following theorem.

Theorem 6.5: Given the topologies as defined above, for $1 \leq q \leq 2 \leq p<\infty$, the following diagram holds

$$
\begin{array}{cc}
G_{p, N} & \rightarrow G_{2, N} \rightarrow G_{q, N} \\
\Downarrow & \Downarrow \\
g_{p, N} \rightarrow & g_{2, N} \rightarrow g_{q, N}
\end{array}
$$

with continuous and dense embeddings.

Proof: The proof is similar to that of Theorem 6.3. For details, see [5].

Again the elements of $g_{q, N}, 1 \leq q<2$, are generalized functionals of white noise, that is, $\mathfrak{g}_{q, N} \subset F\left(S^{*}, \mu\right)$. The next question is, can the above result be extended to $N=\infty$. The estimate on the embedding constant $C_{p, N}$ as given above is not suitable for this. In fact, it diverges to infinity and hence the above result may not extend to $N=\infty$. However one can prove a partial result.

Theorem 6.6: For $1<q \leq 2 \leq p<\infty$

(i) $\quad \mathfrak{g}_{p}=\operatorname{Lim}_{N \rightarrow \infty} \mathfrak{g}_{p, N}, \mathfrak{\varrho}_{q}=\operatorname{Lim}_{N \rightarrow \infty} \mathfrak{\oint}_{q, N}$

(ii) $G_{p}, G_{q}, \mathfrak{g}_{p}, \mathfrak{g}_{q}$ are reflexive Banach spaces,

(iii) $\mathfrak{g}_{p} \Leftrightarrow G_{p}, \mathfrak{g}_{q} \Leftrightarrow G_{q}$ and $G_{p}^{*}=G_{q}, \mathfrak{g}_{p}^{*}=\mathfrak{g}_{q}$.

It is interesting to note that we do not have any inclusion relation between the Banach spaces $G_{p}, G_{q}$ and hence no inclusion relation exists between the corresponding Banach spaces of white noise functionals $\mathcal{G}_{p}, \mathfrak{g}_{q}$. It is easy to verify that $G_{p}$ separates points of $G_{q}$ and hence, by the isomorphism, $\mathfrak{G}_{p}$ separates points of $\varrho_{q}$. For details see [5]. 
Remark 6.7: For $p=q=2$ we obtain the classical Wiener-Ito functionals.

In the spirit of Theorem 4.2, we can construct new generalized functionals from those of Theorem 6.6 by introducing suitable weighting functions other than just the functions of $n$ !. With this end in view, we introduce the following definition.

Definition 6.8: Let $N_{+}$denote the set of nonnegative integers, and $R_{+}$the set of nonnegative real numbers. $A$ function $a: N_{+} \times R \rightarrow R_{+} \cup\{+\infty\}$ is said to be an admissible weighting function if it is a monotone increasing function of both the variables and it satisfies the following properties:

(i) $a(n, s) a(n,-s)=1,($ ii $) a(0, s)=a(n, 0)=1,(i i i) \underset{n, s \rightarrow 0}{\operatorname{Lim}} a(n, s)=1$,

(iv) $\operatorname{Lim}_{s \rightarrow \infty} a(n, s)=\infty, n>1,(v) \operatorname{Lim}_{n \rightarrow \infty} a(n, s)=\infty, s>0$.

Clearly, it follows from $(i)$ and $(i v)$ that $\underset{s \rightarrow \infty}{\operatorname{Lim}} a(n,-s)=0$.

For $p \in[1, \infty]$, define

$$
\Xi_{p} \equiv\left\{\left(K_{0}, K_{1}, K_{2}, \ldots, K_{n}, \ldots\right): K_{n} \in L_{p}\left(I^{n}\right), n \in N_{+}\right\} \equiv \prod_{n=0}^{\infty} L_{p}\left(I^{n}\right)
$$

Clearly this is only a linear vector space (with the product topology but) without any norm topology. Corresponding to $\Xi_{p}$, we can introduce the vector space of white noise functionals

$$
\widehat{\Xi}_{p} \equiv\left\{\left(\phi_{0}\left(K_{0}\right), \phi_{1}\left(K_{1}\right), \phi_{2}\left(K_{2}\right), \ldots, \phi_{n}\left(K_{n}\right), \ldots\right), K \in \Xi_{p}\right\},
$$

where $\left\{\phi_{n}\right\}$ are the elementary homogeneous functionals of white noise as defined in Lemma 6.1 and Lemma 6.2. Being the Cartesian product of infinitely many Banach spaces, these spaces have only the linear structure with the product topology. Thus the map

$$
\left\{K_{n}, n \in N_{+}\right\} \rightarrow\left\{\phi_{n}\left(K_{n}\right), n \in N_{+}\right\}
$$

from $\Xi_{p}$ to $\widehat{\Xi}_{p}$, denoted here by $\Psi$, is merely an algebraic isomorphism.

Now we shall introduce various norm topologies and construct normed spaces so that this becomes an isometry: For $p \in[1, \infty), s \geq 0$, define

$$
G_{p, s} \equiv\left\{K \in \Xi_{p}:\left(\sum_{n=0}^{\infty} n !(a(n, s))^{p}\left\|K_{n}\right\|_{L_{p}\left(I^{n}\right)}^{p}\right)^{1 / p}<\infty\right\} .
$$

Furnished with the norm topology as indicated by the definition, $G_{p, s}$ is a Banach space and for a fixed $p, G_{p, s} \subset G_{p, r}$, for $s>r$. For $1<q \leq 2 \leq p<\infty,\left(G_{p, s}\right)^{*}=G_{q,-s}$. Then we can introduce the generalized functionals of white noise through the isomorphism:

$$
\mathcal{G}_{p, s} \equiv \Psi\left(G_{p, s}\right), \mathcal{G}_{q,-s} \equiv \Psi\left(G_{q,-s}\right)
$$

Theorem 6.9: For $1<q \leq 2 \leq p<\infty$ and $s \in R_{+}$, the following results hold:

(i) $G_{p, s}, G_{q,-s}, \mathcal{G}_{p, s}$, and $\mathfrak{G}_{q,-s}$ are reflexive Banach spaces,

(ii) $\mathcal{G}_{p, s} \Leftrightarrow G_{p, s}, \mathcal{G}_{q,-s} \Leftrightarrow G_{q,-s}$ and $G_{p, s}^{*}=G_{q-s}$, and $\mathcal{G}_{p, s}^{*}=\mathcal{G}_{q,-s}$,

(iii) $\mathcal{G}_{q,-s} \subset F\left(S^{*}, \mu\right)$, are generalized functionals of white noise for $s \geq 0$.

Remark 6.10: For $s=0$, we obtain the results of Theorem 6.6; and, in particular, $\mathfrak{g}_{q,-0}=\mathfrak{g}_{q}$. The family of spaces $\left\{\mathfrak{g}_{q,-s}, s \in R_{+}\right\}$is an expanding family of generalized functionals of white noise more general than those of 'Theorem 6.6 , and they are the duals of the 
Banach spaces $\left\{\mathfrak{G}_{p, s}, s \in R_{+}\right\}$.

The ultimate generalization of this section is given in the following theorem.

Theorem 6.11:

For $0 \leq r \leq s$, the identity map $\mathfrak{G}_{q,-r} \rightarrow \mathfrak{g}_{q,-s}$ is continuous, $\mathfrak{G}_{q} \equiv \bigcup_{r \geq 0} \mathcal{G}_{q,-r}$ is the inductive limit of the spaces $\mathfrak{G}_{q,-r}$ which has the finest locally convex topology for which the embedding $\mathfrak{g}_{q,-r} \rightarrow \mathfrak{g}_{q}$ is continuous. This is the inductive limit topology.

$$
\begin{aligned}
& \mathcal{G}_{q} \text { is the topological dual of } \mathcal{G}_{p} \equiv \bigcap_{r \geq 0} \mathcal{G}_{p, r} \text {; with } \mathcal{G}_{p} \text { having the projective limit } \\
& \text { topology. }
\end{aligned}
$$

The space $\mathfrak{G}_{q} \subset F\left(S^{*}, \mu\right)$ is the space of generalized functionals of Brownian motion and the space $\mathcal{G}_{p}$ is the space of test functionals.

Note that the generalized functionals presented in this section cover those of Nualart and Zakai, [9], and all those presented in the preceding sections.

\section{7. $w^{p, m}$-Based Generalized Functionals of White Noise IV}

In this section we introduce the most general class of generalized functionals that cover all those presented above including the generalized functionals of Hida, Nualart, Zakai, Ustunel and others. Let $W^{p, m, n}$ denote the Sobolev space of functions defined on $I^{n}$ whose distributional derivatives up to order $m$ belong to $L_{p}\left(I^{n}\right)$ with the usual norm topology. The space $W_{0}^{p, m, n}$ is the completion in the above topology of $C^{\infty}\left(I^{n}\right)$ functions having compact supports. The Sobolev space with negative norm denoted by $\mathcal{W}^{q,}-\boldsymbol{m , n}$, where $(1 / p)+(1 / q)=1$, is the dual of $\mathcal{W}_{0}^{p, m, n}$, and it is a class of distributions on $I^{n}$.

Throughout this section, we shall use the hat symbol to distinguish the generalized functionals constructed using Sobolev spaces from those constructed using $L_{p}$ spaces.

Let $1 \leq q \leq 2 \leq p<\infty$ satisfying $p^{-1}+q^{-1}=1$. For each positive number $n$, let $m(n)$ be a real number such that $m(n)>(n / p)$ and consider the Sobolev space $\mathcal{W}_{0}^{p, m(n), n}$ and its dual $W^{q,-m(n), n}$. Recall that $\mathcal{W}_{0}^{p, m, n}$ is the completion in the topology of $\mathcal{W}^{p, m, n}$ of $C^{\infty}$ functions

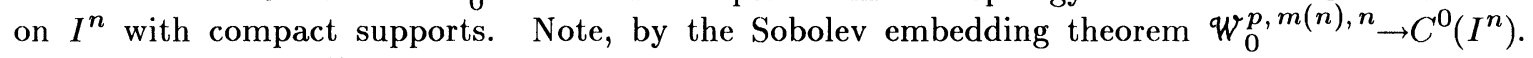
We shall need the following vector spaces. Define

$$
\begin{gathered}
\widehat{G}^{p, m(n), n} \equiv\left\{L \in \widehat{L}_{p}\left(I^{n}\right): L \in W_{0}^{p, m(n), n}\right\} \\
\widehat{G}^{q,-m(n), n} \equiv\left(\widehat{G}^{p, m(n), n}\right)^{*}=\operatorname{dual} \text { of } \widehat{G}^{p, m(n), n} .
\end{gathered}
$$

Furnished with the norm topology

$$
\left\|L_{n}\right\|_{\widehat{G}} p, m(n), n \equiv(n !)^{1 / p}\left\|L_{n}\right\|_{\mathcal{W}^{p, m(n), n}}
$$

the space $\widehat{G}^{p, m(n), n}$ is a Banach space. Its dual is $\widehat{G}^{q,-m(n), n}$ which is furnished with its natural topology given by

$$
\left\|K_{n}\right\|_{\widehat{G}^{q,-m(n), n}} \equiv(n !)^{1 / q}\left\|K_{n}\right\|_{\mathcal{W}^{q,-m(n), n}}
$$

where

$$
\|f\|_{\mathcal{W}^{q,-m(n), n}} \equiv \operatorname{Sup}\left\{|f(\phi)|:\|\phi\|_{\mathcal{W}_{0}^{p, m(n), n}} \leq 1\right\}
$$


Let $\phi_{n}$ denote, as in (6.1), the homogeneous functionals of degree $n$. Define the linear vector space

$$
\widehat{\mathrm{g}}^{p, m(n), n} \equiv\left\{z \in M\left(S^{*}, \mu\right): z=\phi_{n}\left(L_{n}\right), L_{n} \in G^{p, m(n), n}\right\}
$$

which, furnished with the norm topology

$$
\|z\|_{\widehat{\mathcal{G}}^{p, m(n), n}} \equiv\left\|L_{n}\right\|_{\widehat{G}^{p, m(n), n}},
$$

is a Banach space isometrically isomorphic to $\widehat{G}^{p, m(n), n}$. Since $\widehat{G}^{p, m(n), n} \subset \widehat{L}_{p}\left(I^{n}\right) \subset \widehat{L}_{2}\left(I^{n}\right)$ for $p \geq 2$, these are the regular functionals (Wiener-Ito) of white noise. The generalized functionals are the images under $\phi_{n}$ of the Banach space $\widehat{G}^{q,-m(n), n}$. Indeed the following result holds.

Lemma 7.1: For each $K_{n} \in \widehat{G}^{q,-m(n), n},(1 \leq q \leq 2)$, the functional $z^{*} \equiv \phi_{n}\left(K_{n}\right)$ is a well defined generalized random element of $F\left(S^{*}, \mu\right)$.

Proof: The proof is based on Lemma 5.2 and the fact that the completion of $L_{q}\left(I^{n}\right)$ in the topology of $\mathcal{W}^{q,-m(n), n}$ is $\mathcal{W}^{q,-m(n), n}$ itself. For details, see [5, Lemma 5.1].

The class of such functionals denoted by $\widehat{\mathrm{g}}^{q,-m(n), n}$ is given by $\phi_{n}\left(\widehat{G}^{q,-m(n), n}\right)$. We can topologize this vector space by a norm. For each $z^{*} \in \widehat{\mathrm{g}}^{q,-m(n), n}$ we define its norm by

$$
\left\|z^{*}\right\|_{\widehat{\mathfrak{g}}^{q,-m(n), n}} \equiv \operatorname{Sup}\left\{\left|z^{*}(z)\right|,\|z\|_{\widehat{\mathfrak{g}}^{p, m(n), n}} \leq 1, z \in \widehat{\mathfrak{G}}^{p, m(n), n}\right\} .
$$

In this case the natural duality pairing between $z^{*}$ of $\widehat{\mathscr{g}}^{q,-m(n), n}$ and $z$ of $\widehat{\mathcal{g}}^{p, m(n), n}$ is given by

$$
z^{*}(z) \equiv\left\langle z^{*}, z\right\rangle_{\widehat{\mathrm{g}}^{q,-m(n), n}, \hat{\mathrm{G}}^{p, m(n), n}}=\left\langle L_{n}, K_{n}\right\rangle_{\widehat{G}^{q,-m(n), n}, \widehat{G}^{p, m(n), n}},
$$

where $z^{*}=\phi\left(L_{n}\right)$. With this introduction, we can now present the following fundamental result.

Theorem 7.2: For each positive integer $n$, and $1<q \leq 2 \leq p<\infty$ with $p^{-1}+q^{-1}=1$, and $m(n)>(n / p)$, the following results hold:

$$
\begin{aligned}
\widehat{G}^{q,-m(n), n}=\left(\widehat{G}^{p, m(n), n}\right)^{*} \text { and } \widehat{\mathrm{g}}^{q,-m(n), n}=\left(\widehat{\mathrm{g}}^{p, m(n), n}\right)^{*}, \\
\widehat{G}^{p, m(n), n} \rightarrow \widehat{G}^{2,0, n} \rightarrow \widehat{G}^{q,-m(n), n} \\
\widehat{\mathrm{g}}^{p, m(n), n} \rightarrow \widehat{\mathrm{g}}^{2,0, n} \rightarrow \widehat{\mathrm{g}}^{q,-m(n), n}
\end{aligned}
$$

with continuous and dense embeddings.

Proof: For details, see [5].

Remark 7.3A: Note that for $p=q=2$ and $m(n)=(n+1) / 2$, we obtain the generalized functionals due to IIida as given by equation (5.17) of Theorem 5.1.

Remark 7.3B: Since $m(n)>n / p, \mathcal{W}_{0}^{p, m(n), n} \rightarrow C^{0}\left(I^{n}\right)$ and hence one can easily verify that for any $\tau \in I, \quad z^{*} \equiv(\dot{w}(\tau))^{n} \in \widehat{\mathcal{G}}^{q,-m(n), n}$, and, for $z_{n} \in \mathcal{G}^{p, m(n), n}$ with kernel $K_{n}$ we have $\left\langle z^{*}, z_{n}\right\rangle=n ! K_{n}(\tau, \tau, \ldots, \tau)$. Since, by the embedding theorem, $K_{n}$ is continuous, it is clear $\left|\left\langle z^{*}, z_{n}\right\rangle\right|=n !\left|K_{n}(\tau, \tau, \ldots, \tau)\right|<\infty$. Thus $z^{*}$ is a well defined bounded linear functional, while it is not an element of $L_{2}\left(S^{*}, \mu\right)$.

Similar to that in the preceding section, for nonhomogeneous functionals, we introduce the following Sobolev spaces:

Now we consider nonhomogeneous generalized functionals of degree $N \in N_{+}$. Define the 
vector spaces

$$
\begin{gathered}
\widehat{G}_{p, N}^{+} \equiv \prod_{n=0}^{N} \widehat{G}^{p, m(n), n}, 2 \leq p<\infty \\
\widehat{G}_{q, N}^{-} \equiv \prod_{n=0}^{N} \widehat{G}^{q,-m(n), n}, 1 \leq q \leq 2 ;
\end{gathered}
$$

where $m(n)>(n / p)$ for each integer $n$. These vector spaces, furnished with the norm topologies:

$$
\begin{gathered}
\|L\|_{\widehat{G}_{p, N}^{+}} \equiv\left(\sum_{n=0}^{N}\left\|L_{n}\right\|_{\widehat{G}^{p, m(n), n}}^{p}\right)^{1 / p} \\
\|K\|_{\widehat{G}_{q, N}^{-}} \equiv\left(\sum_{n=0}^{N}\left\|K_{n}\right\|_{\widehat{G}^{q,-m(n), n}}^{q}\right)^{1 / q} ;
\end{gathered}
$$

are Banach spaces (reflexive if $q>1)$ and further $\widehat{G}_{q, N}^{-}=\left(\widehat{G}_{p, N}^{+}\right)^{*}$.

Using $\Phi_{N}$ as in the expression (6.10), we introduce

$$
\widehat{\mathrm{g}}_{p, N}^{+} \equiv\left\{z \in F\left(S^{*}, \mu\right): z=\Phi_{N}(L), L \in \widehat{G}_{p, N}^{+}\right\},
$$

for $2 \leq p<\infty$. In this case the dual $\widehat{\mathrm{g}}_{q, N}^{-}$of $\widehat{\mathrm{g}}_{p, N}^{+}$is a very large class of generalized functionals of white noise covering those given by Theorem 5.1 and Theorem 6.4. The following theorem is their generalization [5, Theorem 5.2].

Theorem 7.4: Given the topologies as defined above, for $1<q \leq 2 \leq p<\infty$, the following results hold:

(i) $\widehat{G}_{q, N}^{-}$and $\widehat{\mathrm{g}}_{q, N}^{-}$are the (topological) duals of $\widehat{G}_{p, N}^{+}$and $\widehat{\mathrm{g}}_{p, N}^{+}$respectively.

$$
\begin{aligned}
& \widehat{G}_{p, n}^{+} \rightarrow \widehat{G}_{2, n} \rightarrow \widehat{G}_{q, n}^{-} \\
& \text {\# \# \# } \\
& \widehat{\mathrm{g}}_{p, n}^{+} \rightarrow \widehat{\mathrm{g}}_{2, n} \rightarrow \widehat{\mathrm{g}}_{q, n}^{-},
\end{aligned}
$$

where the injections are continuous and dense.

Proof: See [5, Theorem 5.2].

The following theorem generalizes Theorem 6.6 and it is the limiting version of Theorem 7.4.

Theorem 7.5: For $1<q \leq 2 \leq p<\infty$

(i) $\quad \widehat{\mathrm{g}}_{p}^{+}=\operatorname{Lim}_{N \rightarrow \infty} \widehat{\mathrm{g}}_{p, N}^{+}, \widehat{\mathrm{g}}_{q}^{-}=\operatorname{Lim}_{N \rightarrow \infty} \widehat{\mathrm{g}}_{q, N}^{-}$,

(ii) $\widehat{G}_{p}^{+}, \widehat{G}_{q}^{-}, \widehat{\mathfrak{g}}_{p}^{+}, \widehat{\varrho}_{q}^{-}$are reflexive Banach spaces,

(iii) $\widehat{\mathfrak{G}}_{p}^{+} \Leftrightarrow \widehat{G}_{p}^{+}, \widehat{\mathscr{G}}_{q}^{-} \Leftrightarrow \widehat{G}_{q}^{-}$and $\left(\widehat{G}_{p}^{+}\right)^{*}=\widehat{G}_{q}^{-},\left(\widehat{\mathscr{G}}_{p}^{+}\right)^{*}=\widehat{\mathfrak{G}}_{q}^{-}$. There is no inclusion relation as in (7.12).

Proof: See [5, Theorem 5.3].

For $p \in[2, \infty]$, and $q \in[1,2],(1 / p+1 / q=1)$, introduce the linear vector spaces $\Sigma_{p}$ and $\Sigma_{q}$ as defined by

$$
\Sigma_{p} \equiv\left\{\left(K_{0}, K_{1}, K_{2}, \ldots, K_{n}, \ldots\right): K_{n} \in \mathcal{W}_{0}^{p, m(n), n}, n \in N_{+}\right\} \equiv \prod_{n=0}^{\infty} \mathcal{W}_{0}^{p, m(n), n}
$$




$$
\Sigma_{1} \equiv\left\{\left(L_{0}, L_{1}, L_{2}, \ldots, L_{n}, \ldots\right): L_{n} \in \mathscr{W}^{q,-m(n), n}, n \in N_{+}\right\} \equiv \prod_{n=0}^{\infty} \mathcal{W}^{q,-m(n), n} .
$$

Note that $\Sigma_{q}$ is the algebraic dual of $\Sigma_{p}$ for $1 \leq p<\infty$. We shall use, as in Section 6 , the same symbol $\Psi$ for the algebraic isomorphism between these linear spaces and the universal space $F\left(S^{*}, \mu\right)$ of the white noise functionals. Thus we have

$$
\widehat{\Sigma}_{p} \equiv \Psi\left(\Sigma_{p}\right) \text { and } \widehat{\Sigma}_{q} \equiv \Psi\left(\Sigma_{q}\right)
$$

Now we shall introduce various norm topologies and construct normed spaces so that this becomes an isometry: For $p \in[2, \infty), s \geq 0$, define

$$
\widehat{G}_{p, s} \equiv\left\{K \in \Sigma_{p}:\left(\sum_{n=0}^{\infty} n !(a(n, s))^{p}\left\|K_{n}\right\|_{W_{0}^{p, m(n), n}}^{p}\right)^{1 / p}<\infty\right\} .
$$

Similarly for, $q \in(1,2], s \geq 0$, define

$$
\widehat{G}_{q,-s} \equiv\left\{L \in \Sigma_{q}:\left(\sum_{n=0}^{\infty} n !(a(n,-s))^{q}\left\|L_{n}\right\|_{W_{0}^{q,-m(n), n}}^{q}\right)^{1 / q}<\infty\right\} .
$$

Furnished with the norm topology as indicated by the definition, $\widehat{G}_{p, s}$ is a Banach space and for a fixed $p, \widehat{G}_{p, s} \subset \widehat{G}_{p, r}$, for $s>r$. For $1<q \leq 2 \leq p<\infty,\left(\widehat{G}_{p, s}\right)^{*}=\widehat{G}_{q,-s}$. Then we can introduce the generalized functionals of white noise through the isomorphism:

$$
\widehat{\mathfrak{G}}_{p, s} \equiv \Psi\left(\widehat{G}_{p, s}\right), \widehat{\mathfrak{G}}_{q,-s} \equiv \Psi\left(\widehat{G}_{q,-s}\right)
$$

Theorem 7.6: For $1<q \leq 2 \leq p<\infty$ and $s \in R_{+}$, the following results hold:

(i) $\widehat{G}_{p, s}, \widehat{G}_{q,-s}, \widehat{\mathfrak{G}}_{p, s}$ and $\widehat{\mathrm{G}}_{q,-s}$ are reflexive Banach spaces,

(ii) $\widehat{\mathfrak{G}}_{p, s} \Leftrightarrow \widehat{G}_{p, s}, \widehat{\mathfrak{G}}_{q,-s} \Leftrightarrow \widehat{G}_{q,-s}$ and $\widehat{G}_{p, s}^{*}=\widehat{G}_{q,-s}$, and $\widehat{\mathrm{g}}_{p, s}^{*}=\widehat{\mathfrak{G}}_{q,-s}$.

Remark 7.7: For $s=0$, we obtain the results of Theorem 7.5; and, in particular, $\widehat{\mathrm{g}}_{q,-0}=\widehat{\mathrm{g}}_{q}$. The family of spaces $\left\{\widehat{\mathfrak{g}}_{q,-s}, s \in R_{+}\right\}$is an expanding family of generalized functionals of white noise more general than those of Theorem 7.5, and they are the duals of the Banach spaces $\left\{\widehat{\mathfrak{g}}_{p, s}, s \in R_{+}\right\}$.

The ultimate generalization of this section is given in the following theorem.

\section{Theorem 7.8:}

(1) For $0 \leq r \leq s$, the identity map $\widehat{\mathrm{G}}_{q,-r} \rightarrow \widehat{\mathscr{G}}_{q,-s}$ is continuous.

$\widehat{\mathcal{G}}_{q} \equiv \bigcup_{r \geq 0} \widehat{\mathscr{g}}_{q,-r}$ is the inductive limit of the spaces $\widehat{\mathrm{g}}_{q,-r}$ which has the finest locally convex topology for which the embedding $\widehat{\mathrm{g}}_{q,-r} \rightarrow \widehat{\mathfrak{G}}_{q}$ is continuous.

$\widehat{g}_{q}$ is the topological dual of $\widehat{g}_{p} \equiv \bigcap_{r \geq 0} \widehat{g}_{p, r}$.

The space $\widehat{g}_{q}$ is the space of generalized functionals of Brownian motion and the space $\widehat{g}_{p}$ is the space of test functionals.

Remark 7.9: In view of the preceding results, it is clear that the kernels of homogeneous Brownian functionals can be taken from the space of Schwarz distributions $\mathscr{D}^{*}\left(I^{n}\right)$ giving 
generalized functionals with the corresponding test functionals having kernels from the Schwarz space $\mathscr{D}\left(I^{n}\right)$. This way, one can construct many different classes of locally convex spaces of generalized functionals of Brownian motion. We shall consider this in the future.

\section{Applications}

For lack of space we shall only refer to some applications. The functionals of Brownian motion have many applications in physical and biological sciences. The functionals presented in this paper have found applications in stochastic differential and functional differential equations and systems theory (see [2, 3, 4, 5, 13]), in Malliavin calculus (see [10]), and in stochastic partial differential equations (see Nualart, Zakai [9], Ahmed [5]), in quantum mechanics (see Hida [8]). The problem of synthesis of input-output maps based on optimization in the Hilbert space $L_{2}\left(\Omega, \mathfrak{B}, \mu_{\lambda}\right)$ or equivalently $G_{\lambda}$ for stochastic functional differential equations was studied in [2]. Other natural areas of applications are nonlinear filter and control theory. We present here briefly, an example; for details see [5]. Consider the heat equation with the Neumann boundary condition

$$
\begin{gathered}
\partial u / \partial t=\partial^{2} u / \partial x^{2}, 0 \leq x<\infty, t \geq 0 \\
u(0, x)=0,0 \leq x<\infty \\
\partial u(t, 0) / \partial x=-g(t), t>0
\end{gathered}
$$

where $g(t)=\beta(\nu(t)-u(t, 0))$ for convective heat transfer and $g(t)=\gamma\left(\nu^{4}(t)-u^{4}(t, 0)\right)$ for radiative heat transfer. Here $\beta, \gamma$ are the corresponding heat transfer coefficients and $\nu$ is the temperature of the flame in which the rod is immersed. Due to chaotic nature of the flame, $\nu(t)=m(t)+n(t)$, where $n=\dot{w}$ is the white noise and $m$ is the mean flame temperature. The temperature of the immersed end of the rod is given by the integral equation

$$
u(t, 0)=\int_{0}^{t}(1 / \sqrt{\pi}(t-\tau)) g(\tau) d \tau, t>0
$$

In the case of convective heat transfer we obtain a linear stochastic integral equation for $u(t, 0)$ which contains a term of the form

$$
S(t)=\beta \int_{0}^{t}(1 / \sqrt{\pi}(t-\tau)) \dot{w}(\tau) d \tau
$$

In the case of radiative heat transfer, the corresponding term is given by

$$
S(t) \equiv \int_{I_{t}^{4}}\left(1 / \sqrt{\pi}\left(t-\tau_{1}\right)\right) \delta\left(\tau_{1}-\tau_{2}\right) \delta\left(\tau_{1}-\tau_{3}\right) \delta\left(\tau_{1}-\tau_{4}\right) \prod_{i=1}^{i=4} \dot{w}\left(\tau_{i}\right) d \tau_{i}
$$

where $I_{t}^{4} \equiv[0, t]^{4}$. In the linear case, the kernel $K_{1}(t, \tau) \equiv(1 / \sqrt{\pi}(t-\tau)) \in L_{1}(0, t)$ only for $1 \leq q<2$ and in the nonlinear case, the kernel $K_{4} \in \mathscr{W}^{q,-m, 4}$ for $m>(4(q-1) / q)$. Clearly in both the cases, $S(t)$ is a generalized functional of white noise and belongs to the class considered in Sections 6 and 7 but not in the Hida class (see Section 5). 


\section{References}

[1] Ahmed, N.U., Fourier analysis on Wiener measure space, J. of the Franklin Institute 286, $2,(1968), 143-151$.

[2] Ahmed, N.U., Strong and weak synthesis of nonlinear systems with constraints on the system space $G_{\lambda}$, J. Information and Control, 23, 1, (1973), 71-85.

[3] Ahmed, N.U., Some novel properties of Wiener's canonical expansion, I.E.E.E. Trans. Systems Sci. Cybernetics 5, (1969), 140-144.

[4] Ahmed, N.U., Closure and completeness of Wiener's orthogonal set $G_{n}$ in the class $L_{2}(\Omega, \mathscr{B}, \mu)$ and its applications to stochastic hereditary differential systems, J. Information and Control 17, 2, (1970), 161-174.

[5] Ahmed, N.U., A new class of generalized nonlinear functionals of white noise with applications to random integral equations, Stochastic Analysis and Application 1 (2), (1983), 139-158.

[6] Hida, T., Analysis of Brownian Functionals, Carleton University Math. Lect. Notes, 13, 1978.

[7] Hida, T., Generalized multiple Wiener integrals, Proc. Japan. Acad. 54, ser. A., (1978), 55-58.

[8] Hida, T., Causal calculus of Brownian functionals and its applications, Int. Symp. on Stochastic and Related Topics, Carleton University, Ottawa 1980.

[9] Nualart, D., Zakai, M., Generalized Brownian functionals and the solution to a stochastic partial differential equation, J. of Functional Analysis 84, (1989), 1-18.

[10] Bell, D.R., The Malliavin Calculus, Pitman Monographs \& Surveys in Pure \& Applied Math., 34, Longman Scientific \& Technical, UK, co-publisher: John Wiley, New York 1987.

[11] Wiener, N., The homogeneous chaos, Amer. J. of Math. Vol. LV, (1938), 897-936.

[12] Wiener, N., Nonlinear Problems in Random Theory, The M.I.T. Press, Cambridge, MA 1958.

[13] Dwyer, T.A.W., Analytic evolution equations in Banach spaces, Lect. Notes in Math. Vector Space Measures and Applications 645, Springer-Verlag, Berlin, Heidelberg, New York, (1978), 48-61. 


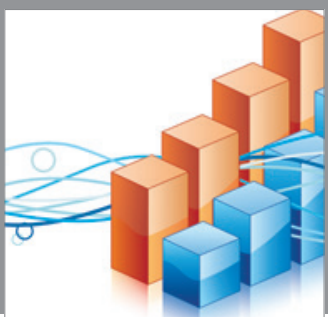

Advances in

Operations Research

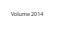

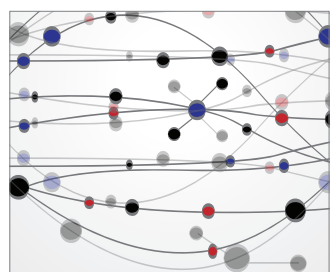

\section{The Scientific} World Journal
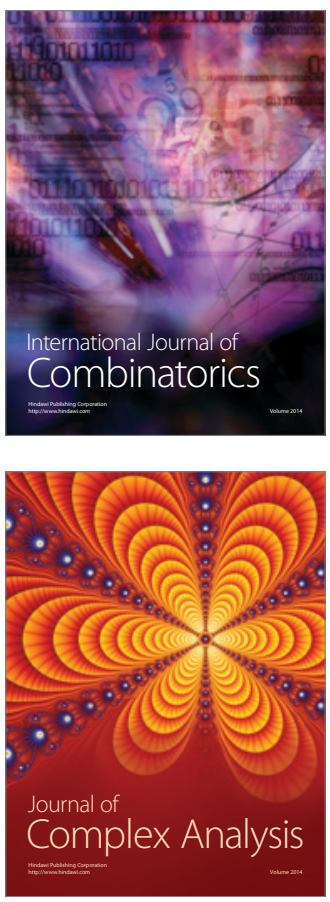

International Journal of

Mathematics and

Mathematical

Sciences
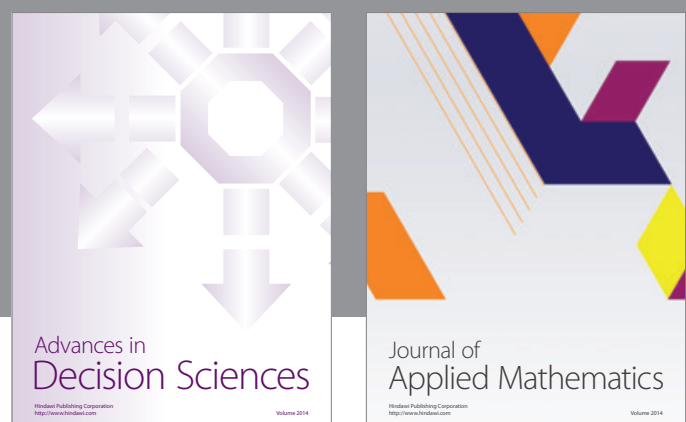

Journal of

Applied Mathematics
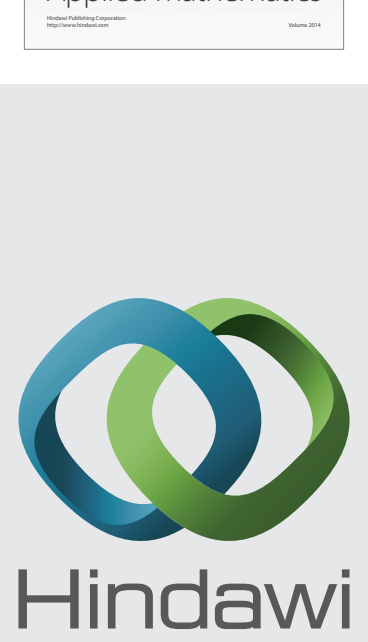

Submit your manuscripts at http://www.hindawi.com
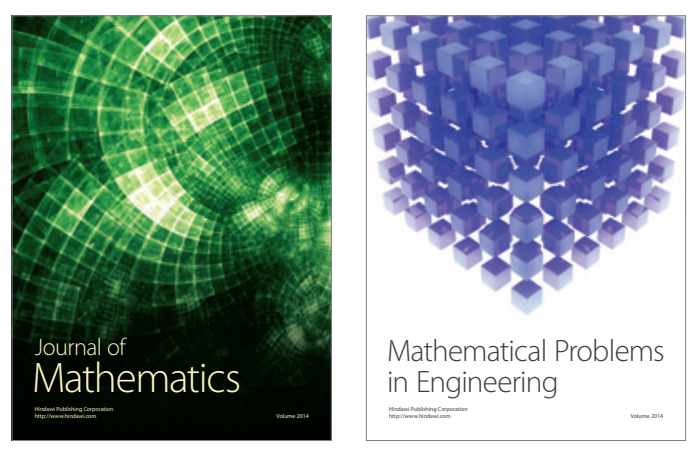

Mathematical Problems in Engineering
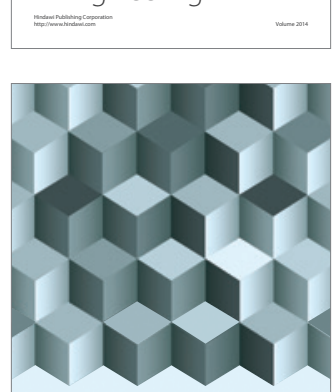

Journal of

Function Spaces
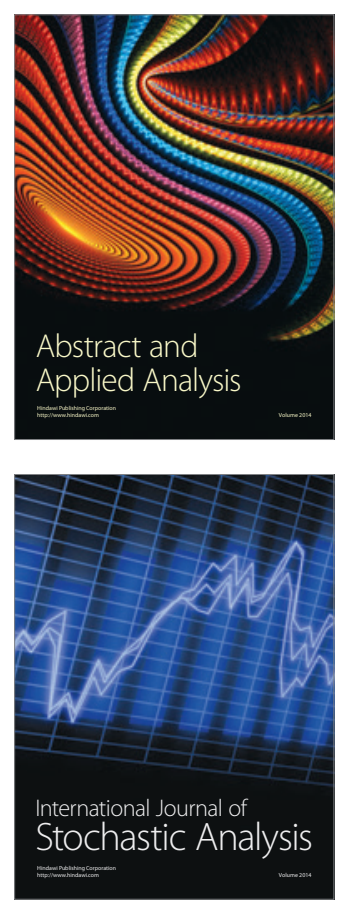

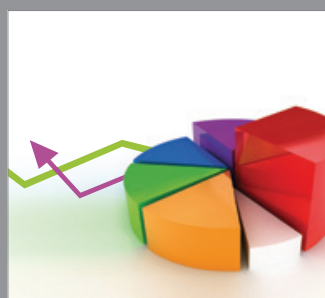

ournal of

Probability and Statistics

Promensencen
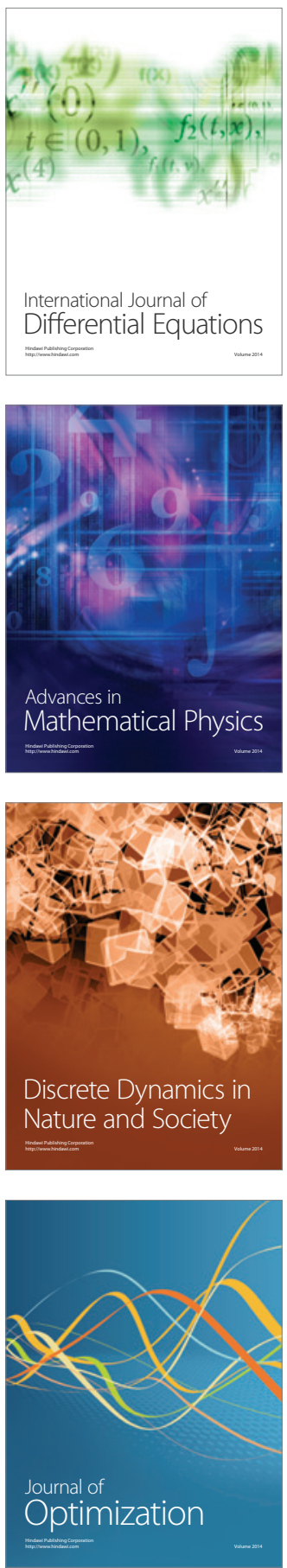